\title{
LA INTUICIÓN INFORMÁTICA: UN ACERCAMIENTO A SU ESTUDIO
}

\author{
Informatics intuition: an approach to it study
}

WALFREDO GONZÁLEZ HERNÁNDEZ*

Recibido: 11 de Noviembre de 2015. Aceptado: 30 de Noviembre de 2015

DOI: http://dx.doi.org/10.21017/rimci.2016.v3.n5.a9

\begin{abstract}
Resumen
El desarrollo de la creatividad en la informática es un imperativo por la importancia que esta tecnología tiene en la sociedad actual. Dentro de los elementos fundamentales para el desarrollo de la creatividad en sentido general uno de los menos tratados en la literatura psicológica y pedagógica lo es sin dudas la intuición. La investigación presenta un acercamiento a esta categoría psicológica y su expresión en la informática para la formación de los profesionales de esta disciplina científica. De la misma manera se expresan las ideas principales sobre su desarrollo durante la enseñanza de la informática.
\end{abstract}

Palabras clave: enseñanza de la informática, creatividad informática, intuición en la informática.

\begin{abstract}
A develop of creativity in informatics science is a must because of the importance that this technology has in today's society. Among the key elements for the development of creativity in general one of the least discussed in the psychological literature and it is undoubtedly intuition. The research presents a psychological approach to this category and its expression in computing for the training of professionals in this field of science. In the same way in paper are expressed develop of intuition principals ideas during informatics teach.
\end{abstract}

Keywords: informatics teach, informatics creativity, informatics intuition.

\section{INTRODUCCIÓN}

L tigüedad hasta nuestros días y muchos supuestos teóricos se han generado desde entonces [1-3]. Los estudios sobre el proceso creativo coinciden en destacar la unidad de la intuición y la lógica, aunque cada teórico ha dado importancia a uno u otro. En el estudio los procesos intuitivos hay un estancamiento que ha durado varios años [4]. Varias corrientes psicológicas han abordado el tema desde diferentes ópticas, destacando en este artículo las tres más relevantes: cognitivismo, el humanismo y el enfoque histórico-cultural.
En la actualidad, la mayoría de los trabajos dedicados a esta temática [5-8], la abordan desde diferentes aristas. Para algunos, [5][7], se aborda como parte de un tipo de pensamiento, de otra manera, [6][9][10], se refieren a la importancia que posee para la toma de decisiones de los directivos. Una de las autoras más reconocidas en la literatura actual, define varios tipos de intuición desde una perspectiva cognitivista [11-13]. En este proceso de desarrollo teórico de los autores consultados no se aprecia la relación de la intuición con la personalidad ni los procesos afectivos que son parte constituyente de ella.

Universidad de Matanzas, Cuba. Correo electrónico: walfredo.glez@umncc.cu 
Sin embargo, en el mundo de hoy se reconoce ampliamente [13-15], el papel de la afectividad en los procesos cognoscitivos que los autores anteriores no abordan desde sus aproximaciones teóricas. Una de las teorías psicológicas que más ha estudiado la relación entre la cognición y lo afectivo es, sin lugar a dudas, el llamado enfoque histórico cultural de Vygostki [16-20]. Dentro de esta teoría se destacan en la actualidad las aproximaciones sobre la subjetividad y su relación con la personalidad [3, 21-23]. A pesar de ser una teoría sobre los procesos psíquicos, en ella no se encuentran referencias teóricas relacionadas con la intuición desde una perspectiva centrada en la personalidad.

De la misma manera han sido pocas las investigaciones relacionadas con la educación de la intuición dentro de un proceso escolarizado [11][24], en estas investigaciones se continúa el sesgo cognitivista que ha sido predominante en las investigaciones psicológicas hasta el momento.

En otro orden de ideas, la informática es hoy una de las ciencias que está marcando pautas en el desarrollo humano y que más ha impactado en este, a través, de su resultado fundamental: las tecnologías informáticas. Por ende la formación de los profesionales de esta ciencia es hoy una necesidad estratégica a nivel mundial. El proceso de formación del ingeniero informático ha sido tratado de manera diferente en la literatura científica [1], pero no ha sido tratada con el proceso creativo y sobre todo el desarrollo de la intuición. Una referencia en este sentido se encuentra en el trabajo de Weigend, en el cual se continúa la presencia de enfoques cognitivistas y sólo aborda la programación [25]. Se ha detectado una referencia al estudio sobre el desarrollo de la intuición en el proceso de formación de estos profesionales [26], sin embargo en este autor no se reconocen las dimensiones e indicadores que pudieran caracterizar este proceso.

Teniendo en cuenta esas consideraciones, este artículo es una aproximación al estudio de la intuición en la informática durante el proceso de formación de ingenieros. Para ello se asumen las posiciones que estudian la subjetividad dentro del enfoque histórico cultural de Vygostki que permita definir la intuición en la informática, sus dimensiones e indicadores así como las vías para su desarrollo.

\section{Desarrollo}

En la literatura consultada de los últimos dos años [27-31], se destacan puntos de contacto, aunque tienen algunos criterios contradictorios. Uno de ellos es la unidad de lo lógico y lo intuitivo. Tomando como base el análisis de estos autores se puede concluir que coinciden en la importancia de la lógica en la etapa de verificación. No es posible la contraposición de la lógica y la intuición por ser etapas de un mismo proceso: la creación. En ese sentido, la intuición plantea nuevas vías de solución a problemas ya conocidos, racionaliza el trabajo pero las ideas generadas en el proceso intuitivo deben ser filtradas utilizando como vía las operaciones y formas lógicas del pensamiento.

Esta «repentina» intuición aparece además en el trabajo científico creador, a veces en las tareas cuya solución hipotética se encuentran más fácilmente, que los métodos o caminos que conducen hacia ella, es decir cuando el resultado, el punto final, al cual evidentemente debe conducir el pensamiento puede anticiparse a pesar de que los caminos que podrían conducir hasta él, todavía no son suficientemente conocidos. Como es conocido estos casos se dan en la ciencia.

El psicoanálisis se ha ocupado preferentemente de la cuestión motivacional de la creación, alegando que esta tiene sus orígenes en los conflictos inconscientes y es una realización de los mismos a través de la sublimación.

Es significativo el análisis de la concepción a partir de la creación de subproductos [10][32]. En el momento de descanso a nivel del subconsciente se van produciendo asociaciones libres, basándose en la experiencia del sujeto, que pasan por una decantación también a este nivel. Estas asociaciones se van agrupando y van conformando una solución del problema que comienza, en este momento, a ser sentida por el individuo como una sensación de bienestar. También resultan interesantes los estudios acerca de las partes del cerebro que son estimuladas en los procesos intuitivos [33]. Cuando este proceso ya está terminando el individuo cree que le han «dictado» la solución del problema. Sin embargo, se reconoce que este proceso no es igual para todos y se conforma en diferentes estilos así como el modo en que aparece,

Rev. Ingeniería, Matemáticas y Ciencias de la Información Vol. 3 / Núm. 5 / enero-junio de 2016; pág. 99-109 
reconociéndose el papel de lo afectivo en el proceso intuitivo [9][34]. En estos autores se aprecia un acercamiento a lo afectivo en el estudio de la intuición que resulta insuficiente para su comprensión y deja varias interrogantes; sin embargo, se destaca lo individual en este proceso.

Es importante destacar en el análisis de la intuición por el cognitivismo las asociaciones libres o divergentes [4] aunque no se ofrezca el modo en que se establecen; la intuición creativa como un estilo que se utiliza en la toma de decisiones [9][10][34-36] como parte integrante de la toma de decisiones o en la resolución de problemas en vez de una función misma. Sinclair plantea «Intuitive expertise draws mostly on locally stored domain-specific patterns accumulated in the past». Esta afirmación relaciona la intuición con la motivación al ser esta importante en el proceso de desarrollo y formación de un experto en un área determinada del conocimiento, sin embargo la autora no lo refiere explícitamente.

Por otro lado, desde una perspectiva histórica cultural se destaca la intuición como parte de los procesos del pensamiento íntimamente relacionados con los procesos afectivos. La intuición se destaca como parte de la relación dialéctica que se establece con el pensamiento lógico, vital para el desarrollo de la creatividad [3]. Aunque no se constata que Vygotsky se haya ocupado de la intuición, se pueden atisbar en su obra elementos que pudieran conducir a explicar el origen de las asociaciones libres expresadas anteriormente.

En la niñez se desarrolla el lenguaje por complejos que se define de la siguiente manera: «El pensamiento en complejos es ya pensamiento coherente y objetivo, aunque no refleja las relaciones objetivas del mismo modo que el pensamiento conceptual»[20], de lo cual se infiere que existen diferencias entre el llamado por el autor pensamiento conceptual. Posteriormente el autor continúa diferenciando estos conceptos.

Un complejo, por lo tanto, es primero y ante todo una agrupación concreta de objetos conectados por vínculos reales, y como no se forma en el plano del pensamiento lógico-abstracto, las uniones que crea, así como las que ayuda a crear, carecen de unidad lógica y pueden ser de muchos tipos diferentes. Cualquier conexión presente puede conducir a la inclusión de un elemento dado en un complejo. La diferencia fundamental entre un complejo y un concepto consiste en lo siguiente: mientras este último agrupa objetos de acuerdo a un atributo, los vínculos que relacionan los elementos de un complejo con el total, y unos con otros, pueden ser tan diversos como en realidad son los contactos y las relaciones de los elementos.» [20]. De este párrafo se puede inferir que existe gran similitud entre las asociaciones divergentes de Sinclair y el pensamiento por complejos abordado por Vygotsky.

Vygotsky, clasifica en 5 tipos al pensamiento complejo en los cuales se aprecian características abordadas de la intuición como son los vínculos variables en la agrupación de los objetos, selección por contraste y semejanza [20]. Otro elemento similar se encuentra en «... El atributo decisivo cambia durante todo el proceso. No existe consistencia en el tipo de enlaces o en la manera en que un eslabón de la cadena se une con el que le precede y el que le sigue, y la muestra original no tiene una significación central. Cada eslabón, una vez incluido en una cadena compleja es tan importante como el primero y puede convertirse en el imán que atraiga a otra serie de objetos.» También de la misma manera se refiere al «...complejo difuso se caracteriza por la fluidez de cada atributo que une los elementos aislados. Por medio de vínculos difusos e indeterminados se forman grupos de objetos o imágenes perceptualmente concretos.» Para este autor entre el pensamiento por complejos y el pensamiento conceptual se encuentra el pensamiento pseudo-conceptual.

Vygotsky afirma «Para completar el esquema del pensamiento en complejos, debemos describir un último tipo, el puente, por decirlo así, entre los descriptos y la etapa final, superior, del desarrollo de la formación del concepto [20]. A este tipo le hemos dado el nombre de pseudo-concepto...»y afirma «En el ambiente experimental, el niño produce un pseudo-concepto cada vez que rodea un ejemplo con objetos que bien podrían haber sido reunidos sobre la base de un concepto abstracto.» De esta manera se va transitando hacia un pensamiento conceptual que, sin embargo, no elimina el pensamiento por complejos sino que este subyace conformando el lenguaje interiorizado. «En el lenguaje interiorizado, el fenómeno llega a 
su punto culminante. Una sola palabra está tan saturada de sentido, que se requerirían muchas otras para explicarla en el lenguaje exterior. No es raro que el lenguaje egocéntrico resulte inexplicable para los demás [20].

«Pero en tanto que en el lenguaje externo el pensamiento está encarnado en palabras, en el lenguaje interiorizado las palabras mueren tan pronto como transmiten el pensamiento. El lenguaje interiorizado es en gran parte un pensamiento de significados puros, es dinámico e inestable, fluctúa entre la palabra y el pensamiento, los dos componentes más o menos delineados del pensamiento verbal. Su verdadera naturaleza y ubicación sólo pueden ser comprendidas después de examinar el siguiente plano del pensamiento verbal, aun más interno que el lenguaje interiorizado... Ese plano es el pensamiento mismo»[20]. Gran parte de los autores dedicados a la intuición lo explican de manera similar, como un proceso del cual se debe tomar nota inmediata para no perder las ideas que se expresan [1][4][9]. La principal limitante de Vygostky, que el autor relaciona con los elementos fundamentales abordados sobre la intuición, está en cómo desarrollarla tomando como base sus ideas.

Por otro lado, el autor no coincide con Vygotsky cuando este expresa que el pensamiento por complejos desaparece y es sustituido, sino que considera que el pensamiento por complejos pasa a formar parte de los procesos no regulados de manera consciente. Ello explica las asociaciones libres que se dan en «períodos de descanso» o en los cuales se «alejan» del problema.

Como se puede apreciar en este bosquejo sobre las concepciones fundamentales acerca de la intuición se trata de un proceso multifactorial con causas que aún no han sido totalmente esclarecidas sobre bases científicas. Se infiere además del bosquejo anterior que es un proceso con un alto grado de incertidumbre que involucra los procesos no conscientes. Es por ello que este autor considera que es un proceso caracterizado por la complejidad que se configura de manera individual en cada individuo.

Por otro lado varios autores reconocen el papel de los procesos afectivos, considerando que el nivel de significatividad emocional es impor- tante para el desarrollo de la intuición y en ello juega un papel fundamental las contradicciones [4][9][11][13]. La investigación reciente en el papel de emociones y experticia también da a entender que la intuición es más contextual de lo que se cree [4][13][37][38], por lo que al considerar la intuición es necesario analizar la actividad en la cual se desarrolla la persona.

Otro elemento a tener en cuenta es la relación intuición y los procesos subjetivos que tiene lugar en la persona. Con base a las referencias bibliográficas seleccionadas, en el análisis de la experiencia se separa lo cognitivo y lo afectivo, considerada como una seria limitante.

En el análisis de la integración de lo cognitivo y lo afectivo se llega a su punto culminante con el concepto de sentido subjetivo [2], el cual se define como la unidad psicológica en desarrollo que integra de forma inseparable procesos simbólicos y emociones, de forma que la emergencia de uno evoca al otro, sin ser su causa, y sin que exista ninguna linealidad en los subsiguientes desdoblamientos de estos procesos, en cuyo curso van apareciendo nuevas funciones psicológicas y nuevos sentidos subjetivos. Los sentidos subjetivos son una producción humana que se desarrolla en la experiencia vivida, pero que toma formas dinámicas de organización, tanto al interior de la personalidad, constituyendo la base para una redefinición de este concepto, como en los diferentes espacios sociales dentro de los cuales se desarrollan las diferentes actividades humanas. Es por ello que, en opinión del autor, puede considerarse la intuición como un proceso que forma parte del sentido subjetivo al desarrollarse sobre la base de la experiencia vivida y adoptando formas dinámicas.

Derivado del párrafo anterior, el autor no coincide con Pretz cuando plantea la existencia de varios tipos de intuición [11]: inferencial, holística y afectiva porque representa una dicotomía del proceso de manera innecesaria, producto de la concepción cognitivista de la autora. Para el autor, la intuición se manifiesta en dependencia de los intereses y motivaciones del individuo constituyendo una expresión de la subjetividad de la persona, concepto, que se manifiesta de diferentes maneras en una actividad determinada [23]. 
Por otro lado es necesario destacar la importancia que juega en el estudio de la intuición el paradigma de la complejidad [39-41]. La complejidad asumida como la superación del paradigma cartesiano de las ciencias conlleva a una compresión holística e integradora de las ciencias [42-44]. En este sentido la creatividad puede ser explicada sobre la base de la solución a la incertidumbre tanto empírica y teórica, mientras más incertidumbre exista en la problemática mayor nivel de intuición es necesaria para su solución. La intuición como proceso marca las pautas en el proceso creativo y declara su dependencia de lo imprevisible y lo no determinista [9][11][13][45-47]. Para otros autores otro elemento para apuntar hacia la complejidad de los estudios de creatividad se encuentra en la subjetividad humana [3] [23] [48].

De la misma manera, para continuar las ideas fundamentales es imprescindible el análisis del paradigma configuracional que se define de la siguiente manera: «... aquellos elementos dinámicos del objeto (sistema de procesos), que tienen una delimitación subjetiva, aunque con un sustento objetivo, se relacionan unos con otros transformándose o interactuando de manera tal, que se integran como un todo en configuraciones de mayor orden, pero no como elementos diferentes que se incorporan, sino como expresiones del todo [49].

En esta definición no se aprecia cuáles son las condiciones necesarias para la aparición de la configuración, así como la manera en que se integran los elementos constituyentes. Además este autor lo delimita en el plano subjetivo, obviando de esta manera que también es configuracional el actuar de los componentes personales y no personales involucrados en el accionar didáctico.

La configuración se puede asumir como «...la organización particular que adoptan sus componentes en un sujeto» para realizar el análisis de la categoría personalidad, sin embargo adolece de ambigüedades en su definición [50]. Por otro lado se ha definido desde el orden psicológico define configuración «... como sistema de formaciones diferentes, donde un mismo elemento psicológico parcial puede aparecer de forma simultánea en unas $\mathrm{u}$ otras de dichas formaciones, incluso con un sentido psicológico diferente [48].»
De esta definición se infieren elementos importantes de la organización de la configuración. Uno de ellos es el sistema de formaciones y se infiere que depende de determinadas situaciones cuando continúa expresando «...la naturaleza configuracional de la personalidad radica en la integración de contenidos psicológicos cognitivos y afectivos en una nueva realidad». Esta última afirmación vincula la configuración a la realidad sin especificar cuáles son los aspectos de la realidad que originan su organización.

En este artículo, considerando los aspectos ya planteados, se define la configuración como la estructura compleja o no en que un sistema complejo organiza o reorganiza a sus componentes (estructurales y/o funcionales) para interactuar con otros sistemas, en dependencia de sus características propias y del otro sistema, así como de la naturaleza de la interacción entre ellos. Se puede aseverar entonces, que un sistema complejo puede adquirir varias configuraciones en su interacción con otros sistemas, permitiéndole interactuar en dependencia de sus condiciones internas y las externas con un máximo de adaptabilidad y flexibilidad. Por ende, todos los sistemas complejos son sistemas configuracionales.

A partir de la variedad de criterios existentes sobre intuición e integrando los elementos ya expuestos para el autor intuición es el proceso complejo configuracional sin regulación consciente que se da al apropiarse de las formas de actividad en dependencia de las formaciones motivacionales del individuo y se basa en experiencias acumuladas para conformar asociaciones libres en un contexto socio histórico determinado que se integra en el sentido subjetivo.

Después de haber definido qué se entiende por intuición es tarea inmediata proceder a esclarecer la relación entre la intuición y la creatividad en la informática. La búsqueda de los elementos necesarios para la consideración de qué es lo creativo y si una persona es creativa o no es hoy en día todavía objeto de fuertes polémicas, más aún en el contexto informático. El proceso creador presente en las personas que se dedican a la solución de problemas informáticos o a su enseñanza presenta características diferentes a otras ciencias ya que está permeado por las características de la rama del saber humano en la cual se ejecuta. El desarrollo 
de la creatividad en un contexto informático se puede desenvolver en tres vertientes fundamentales [51]:

1) Protección de la Información.

2) Transmisión de la Información.

3) Conservación de la Información

El trabajo con los diferentes sistemas informáticos ya creados también es una de las aristas donde se expresa la creatividad del informático, sobre todo cuando estos sistemas conllevan al cambio de paradigma.

En ese sentido, lo novedoso en cuanto a informática se refiere puede encontrarse en el proceso de producción de conceptos, procedimientos, modelos, sistemas y / o algoritmos informáticos que no han sido realizados anteriormente en un contexto social determinado. La novedad en este caso abarca desde los desarrolladores de software, las compañías que marcan pautas en el quehacer informático hasta el estudiante que se prepara para la producción de software o la enseñanza de la informática. Sin embargo, es importante tener en cuenta que el proceso transcurre de manera diferente para cada uno de los casos enunciados anteriormente.

Un elemento a destacar apunta hacia el contexto histórico social en que el individuo se desarrolla, ya que se aborda la problemática del estudiante creador de algoritmos ya descubiertos o no y productor de software, en ocasiones comerciales, hasta el programador que se encuentra en las compañías productoras de software. En el análisis de la creatividad en el contexto de la informática es imprescindible tener en cuenta las condiciones en las cuales se desarrolla y los recursos a disposición de cada uno de ellos que determina la plataforma a utilizar para desarrollar los sistemas. Por ello la consideración de las exigencias sociales es un elemento importante a tener en cuenta en el desarrollo de la creatividad para el contexto de la enseñanza de la informática.

Si bien debe expresarse la integridad de lo cognitivo y lo afectivo, el proceso afectivo en el caso de la informática resulta de vital importancia. La motivación hacia la realización de actividades en torno a la solución de problemas informáticos y las contradicciones que ellos encierran, propicia e induce la realización de las acciones cognoscitivas necesarias para su solución [28-30], [52-55]. Para otros autores la generación, la extensión, la flexibilidad y la autonomía son características esenciales [56].

Por otro lado es necesario caracterizar la actividad informática en su expresión fundamental: el proyecto. En la literatura consultada se han planteado definiciones acerca del proyecto informático [57-60]. Sin embargo, las definiciones trabajadas en la bibliografía no representan la generalidad de las situaciones que pueden encontrarse en la solución de proyectos informáticos por lo que asume la siguiente definición:

Plan de acciones con carácter de sistema, donde se integran las acciones por parte de sus desarrolladores, que contribuyen a la solución de un problema de una esfera determinada del saber humano en la cual se interviene con el uso de, al menos, un Sistema Informático que permita darle solución adecuada y que genere un producto [2].

\section{A. ¿Cuáles son las características en la definición planteada?}

Plan de acciones: Significa que el estudiante o los estudiantes, en Informática los desarrolladores, deben ejecutar un conjunto de acciones para dar solución a su proyecto y que estas acciones tienen un carácter integrador en el cual las soluciones no aparecen aisladas ni fuera del contexto de su trabajo en la solución del proyecto.

Donde se integran acciones por parte de sus desarrolladores: Implica que en la solución del proyecto intervienen la cooperación de sus integrantes en la solución de tareas, en el caso del proyecto en un contexto educativo son los estudiantes, el profesor y los usuarios del sistema; reviste un carácter eminentemente social tanto en la adquisición del conocimiento necesario para su solución como la concatenación de las soluciones aportadas por cada uno en dependencia de sus funciones en la construcción del producto informático.

El problema representa a una esfera del conocimiento humano: La penetración de la Informática en las ramas del saber humano ha planteado a ésta retos en la producción de productos informáticos de calidad y competitivos.

Rev. Ingeniería, Matemáticas y Ciencias de la Información Vol. 3 / Núm. 5 / enero-junio de 2016; pág. 99-109 
Con el uso de, al menos, un sistema Informático: En este caso se aborda la problemática de la utilización de uno o varios sistemas informáticos para su solución puesto que en caso contrario no es un proyecto informático. La optimización de los recursos informáticos en la solución de tareas es muy importante para la satisfacción de las necesidades del cliente en relación con su equipamiento. En el caso de esta tesis se trata de la utilización de un lenguaje de programación para su solución.

Generar un producto: La introducción de la informática en los procesos de producción implica la generación de bienes, en ello los integrantes del proyecto tienen un papel fundamental en su preparación para enfrentar la creación de los productos necesarios para la solución eficiente de la problemática que genera el proyecto.

El proyecto como forma fundamental en la informática está antecedida de una intensa actividad motivacional derivada de las contradicciones expresadas en las situaciones contradictorias que impulsa al individuo a la creación en la informática. La creatividad en la informática se define como el proceso complejo de producción de conceptos, procedimientos, modelos, sistemas y/o algoritmos informáticos relacionados con las vertientes de desarrollo de la informática satisfaciendo las exigencias sociales caracterizadas por la generación, la extensión, la flexibilidad y la autonomía.

La creatividad informática definida anteriormente permite expresar el proceso de creación de un informático teniendo en cuenta como elemento fundamental a la personalidad de un individuo conjuntamente a la atención que debe prestarse al proceso creativo.

Definida la creatividad en la informática, la intuición en la informática debe ser caracterizada en dependencia de la actividad que se realiza como se definió anteriormente. Es por ello que se considera como el proceso complejo sin regulación consciente para conformar asociaciones libres que se da en la apropiación y/u obtención de conceptos, procedimientos, modelos, sistemas y/o algoritmos informáticos, con el proyecto como forma de actividad fundamental y se basa en el sentido subjetivo asociado a la actividad para un contexto socio histórico determinado. Cada uno de estos resultados de la intuición en la actividad informática poste- riormente debe ser verificado lógicamente y, en este sentido, el hardware a utilizar juega un papel esencial como último criterio de veracidad. Es necesario ahondar en este último planteamiento porque, en opinión del autor, la relación dialéctica hardwaresoftware está contextualizada a la situación histórica concreta del problema informático a resolver.

\section{DIMENSIONES E INDICADORES}

Es necesario definir las dimensiones e indicadores que caracterizan la intuición en la informática de tal manera que sea operacionalizable:

\section{A. El proceso complejo configuracional sin regu- lación consciente para conformar asociaciones libres}

1) No hay representación de la secuencia en la obtención de la solución del problema.

2) Expresa la interacción de varios sistemas complejos.

3) Es resultado de diversas interacciones no lineales y no determinísticas.

4) No es posible controlar las condiciones iniciales para que ocurra.

5) Sucede utilizando conexiones entre juicios, conceptos o representaciones en apariencia totalmente diferentes entre sí.

6) Se estructura de manera única cada vez que ocurre.

7) La idea aparece de manera automática de manera integrada.

B. Que se da al apropiación y/u obtención de conceptos, procedimientos, modelos, sistemas y/o algoritmos informáticos

1) Elaborar conceptos, procedimientos, modelos, sistemas y/o algoritmos informáticos para la solución de una problemática.

2) Expresar conceptos, procedimientos, modelos, sistemas y/o algoritmos informáticos en herramientas de desarrollo. 
3) Seleccionar la herramienta más adecuada para el proyecto a ejecutar.

4) Validar conceptos, procedimientos, modelos, sistemas y/o algoritmos informáticos con los métodos elaborados por esta ciencia.

C. Se basa en el sentido subjetivo asociado a la actividad para un contexto socio histórico determinado y aparece como una unidad de contenidos informáticos y procesos afectivos relacionados con este.

1) Se da en la actividad fundamental informática: el proyecto, donde se producen los sentidos subjetivos del área de informática.

2) Aparece relacionado con los motivos que integran la tendencia orientadora de su personalidad.

Desde la perspectiva didáctica diversos autores plantean la necesidad de conocimientos vivenciados y obtenidos con un alto grado de implicación afectiva para que sean tenidos en cuenta por los procesos intuitivos [13][34][47]. Aún no se ha resuelto completamente la problemática planteada sobre el desarrollo de la intuición en la informática como parte de la creatividad informática si no se aborda las vías para su desarrollo. En párrafos anteriores al comentar las ideas de Vygotsky se deja claro que la contradicción es un aspecto esencial en el aprendizaje.

Las contradicciones constituyen la base del desarrollo de la creatividad y en opinión de diversos autores, la enseñanza problémica representa la oportunidad por excelencia para introducirlas en el proceso de enseñanza [61-64]. Se evidencia una fuente de contradicciones importante que puede propiciar la aparición de la intuición puesto que es generadora de motivaciones por el aprendizaje de la informática.

Es necesario entonces caracterizar la enseñanza problémica en la enseñanza de la informática. Dentro del sistema categorial de la enseñanza problémica se destaca la situación contradictoria. La situación contradictoria representa la contradicción que provoca en los estudiantes «sorpresa», un estado de perplejidad ante una situación en la cual él sabe que existe «algo» que está mal, que no es correcto en correspondencia con el sistema de conocimientos del cual se ha apropiado.

La informática como ciencia ha penetrado en las más diversas ramas de la actividad humana desarrollando muchos software para cada una de ellas. Esta es una problemática para su enseñanza puesto que decidir cuál de estos softwares enseñar es una tarea que puede tornarse complicada. Sin embargo, si se asume enseñar los conceptos más generales denominados núcleos conceptuales [52][65] y aquel sistema que los incluya será el seleccionado para su enseñanza. Esta concepción permite una formación más general, holística para la solución de las diversas problemáticas a la cual se enfrenta en su quehacer como informático.

Colocar a los individuos en situaciones que permitan las soluciones con alta variabilidad de asociaciones y formarlos teniendo en cuenta la relación dialéctica entre ellos pudiera ser una solución a esta problemática. Debe tenerse en cuenta además los errores que cometen e incluirlos como parte del aprendizaje, asumiendo que el error es necesario y brindando una formación holística e integral a los individuos.

El enfoque de proyecto propuesto en este trabajo plantea la tarea a solucionar en la cual se integran los conocimientos tomando como base los núcleos conceptuales siendo esta denominada como proyecto con requisitos mínimos. La solución del proyecto con requisitos mínimos implica para el estudiante un conjunto de conocimientos mínimos que deben ser incluidos en el proyecto para que sea evaluado. Sobre la base de estos requisitos mínimos se estructuran las situaciones contradictorias que ha denominado el autor como nodos problémicos asociados al proyecto.

La concatenación de los nodos problémicos y las relaciones que se establecen entre ellos para solucionar el proyecto constituye uno de los aspectos fundamentales del enfoque de sistema en la enseñanza de la Informática [1].

\section{Conclusiones}

La intuición como parte del proceso creador es uno de los elementos menos estudiados en la lite- 
ratura aunque se aprecia un resurgimiento de su análisis en la literatura científica. Variadas definiciones de intuición se encuentra en la literatura de índole cognitivista, sin embargo no ocurre de la misma manera desde el enfoque histórico cultural, referente principal de la investigación. Se toman como punto de partida los resultados enunciados por Vygotsky acerca del pensamiento por complejos llegando a asumir que el pensamiento por complejos no desaparece sino que se mantiene como parte de los procesos no conscientes. En un segundo momento se asume el paradigma de la complejidad. Este paradigma permite explicar las características de no linealidad de los procesos intuitivos así como la variabilidad de los factores necesarios para que ocurra. También permite definir el concepto de configuración sobre la base de la interrelación de dos sistemas complejos. La sistematización de estos fundamentos teóricos permite definir la intuición desde una perspectiva histórica y cultural.

A continuación se aborda el desarrollo de la informática como ciencia y su forma de organización fundamental de la actividad: el proyecto. Este análisis de la informática permite definir el concepto de intuición en la informática sobre la base de los resultados fundamentales de la actividad informática. Se obtienen de este análisis teórico las dimensiones e indicadores que permiten operacionalizar esta definición.

Por último, se realiza un análisis de la enseñanza de la informática así como sus enfoques actuales. Se fundamenta cómo la integración de los enfoques de sistema, el enfoque problémico y el enfoque de proyectos es una de las vías para el desarrollo.

\section{REFERENCIAS}

[1] W. González, «Metodología para contribuir al desarrollo de la creatividad en estudiantes de la educación superior a través de la enseñanza de la programación.» Academia de Ciencias de Cuba. Universidad Pedagógica «Enrique José Varona», 2004.

[2] W. González, Creativity Development in Informatics Teaching Using the Project Focus, IJEP., vol. 3. 2013.

[3] A. Mitjáns, «Subjetividad, Complejidad y Educación.» Psicología para América Latina, 2010.
[4] M. Sinclair, «Intuitive profile of makers.» Academy of Management annual meeting San Antonio, TX, August, 12-16.

[5] L. Dorfman and A. Butakova, Personality intuition type and divergent thinking, Proceedings of the Twenty-third Biennial Congress of the International Association of Empirical Aesthetics. New York, NY, USA: In A. Kozbelt (Ed.), 2014.

[6] O. Hyppänen, «Decision Makers' Use of Intuition at the Front End of Innovation.» Aalto University, 2013.

[7] A. M. Rivers, «Shifting goals for unconscious thinkers?: using reevaluation to test between fuzzy intuition and an active unconscious.» Montana State University, 2013.

[8] A. Woolley and O. Kostopoulou, Clinical intuition in family medicine: more than first impressions, vol. 11. The Annals of Family Medicine, 2013.

[9] E. Dane and M. Pratt, «Exploring intuition and its role in managerial decision making," Academy of Management Review, vol. 32, no. 1, pp. 33-54, 2007.

[10] M. Sinclair and M. Ashkanasy, Intuition: Myth or a Decision-making Tool?. Management Learning, 2005.

[11] J. Pretz, Intuition versus analysis: Strategy and experience in complex everyday problem solving, vol. 3. Memory and Cognition, 2008.

[12] J. Pretz, «Types of intuition: inferential and holistic.» UK: Edward Elgar Publishing Limited, 2011.

[13] J. E. Pretz and K. S. Totz, Measuring individual differences in affective, heuristic, and holistic intuition. Personality and Individual Differences, 2007.

[14] T. Betsch, Preference theory: an affect-based approach to recurrent decision making, In B. S. Haberstroh (Ed.), The routines of decision making. Mahwah, NJ: Lawrence Erlbaum Associates, 2005.

[15] E. G Fernández y M. D. Díaz, «Affective Induction and Creative Thinking,» Creativity Research Journal, vol. 25, no. 2, pp. 213-221, 2013.

[16] M. Mukute, «Cultural Historical Activity Theory, Expansive Learning and Agency in Permaculture Workplaces.» Southern African Journal of Environmental Education, 26, 2009.

[17] W. Roth and Y. Lee, «'Vygotsky's neglected legacy’: Cultural-historical activity theory.,» Review of Educational Research, vol.77, no. 2, pp. 186-232, 2007.

[18] A. Stetsenko and I. M. Arievitch, The Selfin Cultural-Historical Activity Theory Reclaiming the Unity of Social and Individual Dimensions of Human Development, Theory \& Psychology., vol. 4. 2004. 
[19] N. Talizina, Y. Solovieva and L. Q. Rojas, «Aproximación de la actividad en psicología y su relación con el enfoque histórico-cultural de L. S. Vigotsky.» Novedades Educativas, 30, 2010.

[20] L. S. Vygotsky, Pensamiento y lenguaje. Teoría del desarrollo cultural de las funciones psíquicas. Argentina: Ediciones Fausto, 1995.

[21] A. Díaz and F. González R, Subjetividad: una perspectiva histórico cultural, Conversación con el psicólogo cubano Fernando González Rey., vol. 3. Universitas Psychologica, 2005.

[22] O. González, «La subjetividad desde la perspectiva histórico-cultural: un tránsito desde el pensamiento dialéctico al pensamiento complejo,» Revista Colombiana de Psicología, 17, 2008.

[23] F. González, «Las categorías de sentido, sentido personal y sentido subjetivo en una perspectiva histórico-cultural: un camino hacia una nueva definición de subjetividad,» Universitas Psychologica, vol. 9, no. 1, pp. 241-253, 2010.

[24] R. Hersh, Mathematical Intuition: Poincaré, Pólya, Dewey The Courant-Friedrichs-Lewy (CFL) Condition. Springer.

[25] M. Weigend, From intuition to programme Informatics Education-The Bridge between Using and Understanding Computers, Springer. 2006.

[26] W. González, Intuition as Part of Informatics Creativity, IJEP, vol. 7. 2013.

[27] J. V. Bittner and H. Heidemeier, «Competitive mindsets, creativity, and the role of regulatory focus. Thinking Skills and Creativity,» vol. 9, no. 0, pp. 59-68, 2013.

[28] M. Kilgour, S. Sasser and S. Koslow, «Creativity Awards: Great Expectations?,» Creativity Research Journal, vol. 25, no. 2, pp. 163-171, 2013.

[29] A. J. O'Connor, C. J. Nemeth and S. Akutsu, "Consequences of Beliefs about the Malleability of Creativity.,» Creativity Research Journal, vol. 25, no. 2, pp. 155-162, 2013.

[30] H. Pluut and P. L. Cur ${ }^{\circ} e u$, «The role of diversity of life experiences in fostering collaborative creativity in demographically diverse student groups, Thinking Skills and Creativity, vol. 9, no. 0, pp. 1623, 2013.

[31] Y. Yeh and Y. Chen, «From knowledge sharing to knowledge creation: A blended knowledgemanagement model for improving university students' creativity,» Thinking Skills and Creativity, vol. 7, no. 3, pp. 245-257, 2012.

[32] H. Mintzberg, B. Ahlstrand and J. Lampel, Strategy safari: A guided tour through the wilds of strategic management. New York: Free Press, 1998.
[33] M. Reimann and A. Bechara, «The somatic marker framework as a neurological theory of decisionmaking: Review, conceptual comparisons, and future neuroeconomics research, Journal of Economic Psychology, vol. 31, no. 5, pp. 767-776, 2010.

[34] M. Sinclair, N. M. Ashkanasy, P. Chattopadhya and M. V. Boyle, «Determinants of intuitive decision - making in management: The moderating role of aff ect. In N. M.» 2002.

[35] C. Morewedge and D. Kahneman, «Associative processes in intuitive judgment,» Trends in Cognitive Sciences, vol. 14, no. 10, 2010.

[36] S. Epstein, «Intuition from the Perspective of Cognitive-Experiential Self-Theory. In: Intuition in judgment and decision making,» Libr. Congr. Cat.Publ.Data N. Y. USA, pp. 3-22.

[37] A. L. Baylor, «A U- shaped model for the development of intuition by level of expertise.» New Ideas in Psychology, 2001.

[38] J. F. Coget, «Leadership in motion: An investigation into the psychological processes that drive behavior when leaders respond to 'real- time' operational challenges,» Doctoral dissertation, Anderson School of Management, UCLA, 2004.

[39] C. S. Bertuglia and F. Vaio, Nonlinearity, Chaos, and Complexity. New York USA: Oxford University Press Inc, 2005.

[40] A. Dijksterhuis and H. Aarts, «Goals, attention, and (un) consciousness.» Annual Review of Psychology, 61: 467-490, 2010.

[41] W. B. Richardson, Discovering Complexity, Decomposition and Localization as Strategies in Scientific Research. London, England: The MIT Press, 2010.

[42] M. Almeida, «Para comprender la complejidad.» Hermosillo, Sonora, MÉXICO Multiversidad Mundo Real Edgar Morin, A.C., 2008.

[43] T. Jörg, New Thinking in Complexity for the Social Sciences and Humanities a Generative, Transdisciplinary Approach. The Netherlands: Springer Dordrecht Heidelberg, 2010.

[44] R. C. Stacey, Strategic management and organisational dynamics: The challenge of complexity to ways of thinking about organisations., 6th ed. England: Pearson Education Limited Edinburgh Gate, 2011.

[45] F. T. Arecchi, Dynamics of consciousness: Complexity and creativity, Psychophysiology, vol. 2. 2010.

[46] E. Dane, Capturing intuitions «in fl ight»: observations from research on attention and mindfulness In $M$, Sinclair (Ed.), Handbook of intuition research. UK: Edward Elgar Publishing Limited, 2011.

Rev. Ingeniería, Matemáticas y Ciencias de la Información Vol. 3 / Núm. 5 / enero-junio de 2016; pág. 99-109 
[47] M. Sinclair, An integrated framework of intuition, Handbook of intuition research. UK: Edward Elgar Publishing Limited, 2011.

[48] F. González, «L. S. Vygotski y el problema de la personalidad en el enfoque histórico-cultural,» presented at the III Conferência de Pesquisa Sóciocultural, Campinas, Sâo Paulo, 2000.

[49] H. Fuentes and I. Álvarez, «Introducción a la didáctica la conducción del proceso de enseñanza aprendizaje en la educación superior.» Santiago de Cuba: Pueblo y Educación.

[50] Colectivo de Autores, «Pensando en la personalidad (L. F. Rius Ed.).» Ciudad de la Habana: Editorial Félix Varela, 2003.

[51] C. Expósito, «Elementos de metodología de la enseñanza de la informática.» Editorial Pueblo y Educación. Cuba, 2009.

[52] W. González and V. Estrada, «El desarrollo de la creatividad en la enseñanza de la informática a través del enfoque de proyectos.,» COMPUMAT, Ciudad de la Habana, 2014.

[53] W. Lin, K. Hsu, H. Chen and W. Chang, «Different attentional traits, different creativities,» presented at the Thinking Skills and Creativity, 2013, vol. 9, pp. 96-106.

[54] P. Merrotsy, «Tolerance of Ambiguity: A Trait of the Creative Personality?,» presented at the Creativity Research Journal, 2013, vol. 25, pp. 232237.

[55] E. M. Sayed and A. H. Mohamed, «Gender Differences in Divergent Thinking: Use of the Test of Creative Thinking-Drawing Production on an Egyptian Sample.,» presented at the Creativity Research Journal, 2013, vol. 25, pp. 222-227.

[56] M. Djikic, K. Oatley and M. C. Moldoveanu, «Opening the Closed Mind: The Effect of Exposure to Literature on the Need for Closure,» presented at the Creativity Research Journal, 2013, vol. 25, pp. 149-154.
[57] I. Ivan, C. Ciurea, B. Vintilă and G. Noşca, Particularities of Verification Processes for Distributed Informatics Applications, vol. 17. Informatica Economica, 2013.

[58] C. A. Kulikowski, «The 50th Anniversary IMIA History of Medical Informatics Project.» Acta Informatica Medica, 22(1), 68, 2014.

[59] M. E. F. Parker and Y. F. del Monte, The Agile Management of Development Projects of Software Combining Scrum, Kanban and Expert Consultation Open Source Software: Mobile Open Source Technologies. Springer, 2014.

[60] Y. Zulueta, E. Despaigne and A. Hernández, «La gestión de riesgos en la producción de software y la formación de profesionales de la informática: experiencias de una universidad cubana,» REICIS: Revista Española de Innovación, Calidad e Ingeniería del Software, vol. 5, No. 3, 2013.

[61] L. Betancourt and O. Londoño, «Acerca de las ideas, la creatividad y los problemas en la producción de ciencia.» 2013.

[62] C. Burnett, «Holistic approaches to creative problem solving," A thesis submitted in conformity with the requirements for the degree of Doctor of Education, University of Toronto, Canadá, 2011.

[63] G. Molnár, S. Greiff and B. Csapó, «Inductive reasoning, domain specific and complex problem solving: Relations and development,» presented at the Thinking Skills and Creativity, 9(0), 2013, pp. 35-45.

[64] Á. P. Velázquez, M. H. Pérez and Y. Rodríguez, «Elementos teóricos de la enseñanza problémica. Métodos y Categorías.,» Gaceta Médica Espirituana, vol. 14, pp. 34-50, 2012.

[65] W. González, V. Estrada and M. Martínez, «El enfoque de sistema en la enseñanza de la Informática para el desarrollo de la creatividad,» Revista Enseñanza Universitaria, vol. 32, pp. 45-56, 2006. 
